\title{
Strings on semisymmetric superspaces
}

\section{K. Zarembo ${ }^{1}$}

CNRS - Laboratoire de Physique Théorique, École Normale Supérieure, 24 rue Lhomond, 75231 Paris, France

Department of Physics and Astronomy, Uppsala University, SE-751 08 Uppsala, Sweden

E-mail: Konstantin.Zarembo@lpt.ens.fr

ABSTRACT: Several string backgrounds which arise in the AdS/CFT correspondence are described by integrable sigma-models. Their target space is always a $\mathbb{Z}_{4}$ supercoset (a semi-symmetric superspace). Here we list all semi-symmetric cosets which have zero beta function and central charge $c \leqslant 26$ at one loop in perturbation theory.

Keywords: Sigma Models, AdS-CFT Correspondence, Integrable Field Theories

ARXIV EPRINT: 1003.0465

\footnotetext{
${ }^{1}$ Also at ITEP, Moscow, Russia.
} 


\section{Contents}

1 Introduction 1

2 Sigma model 2

3 Beta function and central charge $\quad 5$

4 Conformal sigma models $\quad 8$

5 String sigma-models $\quad 11$

6 Conclusions 13

A Rank of kappa symmetry 14

$\begin{array}{lll}\text { A.1 Type- } U 1 & 15\end{array}$

$\begin{array}{lll}\text { A.2 Type- } U 2 & 17\end{array}$

$\begin{array}{lll}\text { A.3 Type- } U 3 & 17\end{array}$

$\begin{array}{llr}\text { A.4 Type- } U 4 & 18\end{array}$

$\begin{array}{llr}\text { A.5 Type-O1 } & 19\end{array}$

$\begin{array}{lll}\text { A.6 } & \text { Type-O2 } 20\end{array}$

$\begin{array}{lll}\text { A.7 Type-Tu } & 21\end{array}$

A.8 Type-To 22

\section{Introduction}

A semi-symmetric superspace is a coset of a supergroup which possesses an additional $\mathbb{Z}_{4}$ symmetry [1], thus generalizing the notion of ordinary, $\mathbb{Z}_{2}$ invariant symmetric space. Sigma-models on semi-symmetric superspaces possess a number of interesting properties. Perhaps the main motivation to study them comes from the AdS/CFT duality. The holographic duals of superconformal field theories in diverse dimensions are string theories on Anti-de-Sitter backgrounds with Ramond-Ramond fluxes. In many cases (and certainly in all maximally symmetric cases), the worldsheet sigma-models on such backgrounds are $\mathbb{Z}_{4}$ cosets [2]. The best known example is the Green-Schwarz string action on $A d S_{5} \times S^{5}$ [3], which is a $\mathbb{Z}_{4}$ coset of $\operatorname{PSU}(2,2 \mid 4)$, the superconformal group in four dimensions. ${ }^{1}$ One can define a Green-Schwarz-type sigma-model on any $\mathbb{Z}_{4}$ coset. The $\mathbb{Z}_{4}$ symmetry plays a crucial role in this construction by yielding the fermionic Wess-Zumino term in the sigmamodel action [2].

A remarkable property of the Green-Schwarz-type $\mathbb{Z}_{4}$ cosets is their classical integrability [5], which parallels integrability of bosonic symmetric-space sigma-models [6]. A Lax

\footnotetext{
${ }^{1}$ The manifestly $\mathbb{Z}_{4}$-invariant form of the Metsaev-Tseytlin action is given in [4].
} 
representation of the equations of motion in semi-symmetric cosets can be constructed using the $\mathbb{Z}_{4}$ symmetry in a uniform, purely algebraic way. ${ }^{2}$ Perhaps this is why integrability arises in the AdS/CFT correspondence.

All semi-symmetric superspaces are classified [1], and one can scan the list of the $\mathbb{Z}_{4}$ cosets for potentially interesting integrable models, in particular for integrable string backgrounds. To be a string background, a $\mathbb{Z}_{4}$ coset must satisfy two additional conditions: its beta function should vanish and it should have central charge $c=26$.

After reviewing the construction of the Green-Schwarz-type sigma-model on a semisymmetric superspace, we will compute its beta function and central charge at one loop following [7-9]. Then we will list all cosets that satisfy the beta-function and the central charge constraints.

\section{Sigma model}

A coset $G / H_{0}$ of a supergroup $G$ is a semi-symmetric superspace if it is invariant under a $\mathbb{Z}_{4}$ symmetry, generated by a linear automorphism $\Omega$ of the Lie algebra of $G$, $\Omega: \mathfrak{g} \rightarrow \mathfrak{g}, \Omega([X, Y])=[\Omega(X), \Omega(Y)], \Omega^{4}=\mathrm{id}$. The superalgebra $\mathfrak{g}$ then admits a $\mathbb{Z}_{4}$ decomposition:

$$
\mathfrak{g}=\mathfrak{h}_{0} \oplus \mathfrak{h}_{1} \oplus \mathfrak{h}_{2} \oplus \mathfrak{h}_{3},
$$

which is consistent with the (anti-)commutation relations: $\left[\mathfrak{h}_{n}, \mathfrak{h}_{m}\right\} \subset \mathfrak{h}_{(n+m) \bmod 4}$. The subspace $\mathfrak{h}_{n}$ consists of the elements of $\mathfrak{g}$ with the $\mathbb{Z}_{4}$ charge $n$ :

$$
\Omega\left(\mathfrak{h}_{n}\right)=i^{n} \mathfrak{h}_{n} .
$$

The denominator subalgebra of a semi-symmetric coset is the $\mathbb{Z}_{4}$-invariant subspace $\mathfrak{h}_{0}$. The fermion number $F$ is the $\mathbb{Z}_{4}$ charge $\bmod 2$ : the bosonic subalgebra of $\mathfrak{g}$ is $\mathfrak{h}_{0} \oplus \mathfrak{h}_{2}$ and all of the odd generators belong to either $\mathfrak{h}_{1}$ or $\mathfrak{h}_{3}$.

The worldsheet embedding in $G / H_{0}$ is parameterized by a coset representative $g(x) \in$ $G$, subject to gauge transformations $g(x) \rightarrow g(x) h(x)$ with $h(x) \in H_{0}$. The global $G$-valued transformations act on $g(x)$ from the left: $g(x) \rightarrow g^{\prime} g(x)$. The action of the sigma-model can be written in terms of the $\mathbb{Z}_{4}$ decomposition of the left-invariant current $g^{-1} \partial_{\mu} g$ :

$$
J_{\mu}=g^{-1} \partial_{\mu} g=J_{\mu 0}+J_{\mu 1}+J_{\mu 2}+J_{\mu 3} .
$$

The $\mathfrak{h}_{0}$ component of the current transforms as a connection under gauge transformations: $J_{\mu} \rightarrow h^{-1} J_{\mu 0} h+h^{-1} \partial_{\mu} h$. The other three components transform as matter fields in the adjoint: $J_{\mu 1,2,3} \rightarrow h^{-1} J_{\mu 1,2,3} h$.

The action must be gauge invariant and $\mathbb{Z}_{4}$-symmetric. By power counting, the only possible terms ${ }^{3}$ are $J_{2} J_{2}$ and $J_{1} J_{3}$. A particularly interesting case, and the one that we

\footnotetext{
${ }^{2}$ The original construction of [5] for $\mathrm{PSU}(2,2 \mid 4) / \mathrm{SO}(4,1) \times \mathrm{SO}(5)$ relies only on the $\mathbb{Z}_{4}$ decomposition of the symmetry algebra and thus applies to any semi-symmetric coset.

${ }^{3}$ These terms describe coupling to the metric and to the RR fields. In certain cases it should be possible to switch on the B-field (the theta-term in the sigma-model action) or its field strength (the bosonic WessZumino term). For example, if the denominator of the coset contains a U(1) factor, it is possible to add a theta-term $i \vartheta \varepsilon^{\mu \nu} \partial_{\mu} J_{\nu 0}^{\mathrm{U}(1)}$.
} 
will consider here is ${ }^{4}$

$$
S=\frac{1}{2 \kappa^{2}} \int d^{2} x \operatorname{Str}\left(\sqrt{h} h^{\mu \nu} J_{\mu 2} J_{\nu 2}+i \varepsilon^{\mu \nu} J_{\mu 1} J_{\nu 3}\right) .
$$

The "supertrace" $\operatorname{Str}(\cdot \cdot)$ denotes the $G$ and $\mathbb{Z}_{4}$ invariant bilinear form on $\mathfrak{g}$, and $\kappa$ is the sigma-model coupling $\left(\kappa^{2}=2 \pi \alpha^{\prime} / R^{2}\right.$, where $R$ is the radius of $\left.G / H_{0}\right)$. The equations of motion for this action admit a Lax representation [5] making the world-sheet sigma-model classically integrable.

The expansion of the Lagrangian in (2.4) around $g=1$ (the flat-space limit) has the form $\partial X \partial X+\bar{\theta} \partial X \partial \theta$ typical for the Green-Schwarz superstring [10]. And indeed the Green-Schwarz action on many AdS backgrounds can be described as (2.4) for various $\mathbb{Z}_{4}$ cosets $[3,8,9,11-20]$. Just like the ordinary Green-Schwarz action, (2.4) may possess local fermionic kappa-symmetries which, in effect, means that some of the fermion dimensions are unphysical and have to be removed by an appropriate gauge fixing prior to quantization. The rank of the kappa-symmetry depends on the structure of the coset and will be computed in section 4 for all sigma-models with the vanishing one-loop beta-function.

To illustrate these points and to set up the stage for the subsequent one-loop calculations, let us expand the action (2.4) around an arbitrary bosonic background ${ }^{5} \bar{g}(x)$, introducing the following notations for the background currents:

$$
\begin{aligned}
& \left(\bar{g}^{-1} \partial_{\mu} \bar{g}\right)_{0}=A_{\mu}, \\
& \left(\bar{g}^{-1} \partial_{\mu} \bar{g}\right)_{2}=K_{\mu} .
\end{aligned}
$$

Here $A_{\mu}$ is the background gauge field. We will denote by $D_{\mu}$ the background covariant derivative: $D_{\mu}=\partial_{\mu}+\left[A_{\mu}, \cdot\right]$, and by $F_{\mu \nu}$ the background field strength: $F_{\mu \nu}=\partial_{\mu} A_{\nu}-$ $\partial_{\nu} A_{\mu}+\left[A_{\mu}, A_{\nu}\right]$. The currents $A_{\mu}$ and $K_{\mu}$ are assumed to satisfy the classical equations of motion:

$$
\begin{aligned}
{\left[K_{\mu}, K_{\nu}\right]+F_{\mu \nu} } & =0 \\
D_{\mu} K_{\nu}-D_{\nu} K_{\mu} & =0 \\
\nabla_{\mu} K^{\mu} & =0
\end{aligned}
$$

where $\nabla_{\mu} K^{\nu}=D_{\mu} K^{\nu}+\Gamma_{\mu \lambda}^{\nu} K^{\lambda}$ and $\Gamma_{\mu \lambda}^{\nu}$ are the Christoffel symbols of the worldsheet metric. The first two equations are identities that follow from the flatness of the current $\bar{g}^{-1} \partial_{\mu} \bar{g}$. The equations of motion for the metric are the Virasoro constraints:

$$
h^{\mu \nu} \operatorname{Str} K_{ \pm \mu} K_{ \pm \nu}=0
$$

where $K_{ \pm \mu}$ are the chiral components of $K_{\mu}$ :

$$
K_{ \pm \mu}=\frac{1}{2}\left(\delta_{\mu}^{\nu} \pm \frac{i}{\sqrt{h}} h_{\mu \lambda} \varepsilon^{\lambda \nu}\right) K_{\nu}
$$

\footnotetext{
${ }^{4}$ We consider the Euclidean worldsheet, which is why the second term in the Lagrangian is multiplied by $i$. After the Wick rotation the action becomes real.

${ }^{5}$ The background-field calculations have been done for the Green-Schwarz-type cosets [8], as well as for many related pure-spinor type sigma-models [2, 9, 21-24].
} 
In order to expand around the classical background $\bar{g}(x)$ we choose the coset representative in the form

$$
g=\bar{g} \mathrm{e}^{\kappa X},
$$

where $X \in \mathfrak{h}_{1} \oplus \mathfrak{h}_{2} \oplus \mathfrak{h}_{3}$. Under gauge transformation that also act on the background field: $\bar{g} \rightarrow \bar{g} h, X$ transforms in the adjoin: $X \rightarrow h^{-1} X h$. It is straightforward to plug the coset representative (2.9) into the action and expand the latter in the powers of the coupling $\kappa$. The current (2.3) expands as

$$
J_{\mu}=A_{\mu}+K_{\mu}+\frac{1-\mathrm{e}^{-\kappa \mathrm{ad} X}}{\operatorname{ad} X} \mathcal{D}_{\mu} X=A_{\mu}+K_{\mu}+\kappa \mathcal{D}_{\mu} X-\frac{\kappa^{2}}{2}\left[X, \mathcal{D}_{\mu} X\right]+\cdots,
$$

where the long derivative $\mathcal{D}_{\mu}$ is defined by

$$
\mathcal{D}_{\mu}=\partial_{\mu}+\left[\bar{g}^{-1} \partial_{\mu} \bar{g}, \cdot\right]=D_{\mu}+\left[K_{\mu}, \cdot\right] .
$$

Unlike the covariant derivative $D_{\mu}$, which commutes with the $\mathbb{Z}_{4}$ grading, the long derivative $\mathcal{D}_{\mu}$ does not have definite $\mathbb{Z}_{4}$ charge. Thus, $\left(D_{\mu} X\right)_{n}=D_{\mu} X_{n}$ for any $n$ and $\left(\mathcal{D}_{\mu} X\right)_{2}=$ $D_{\mu} X_{2}$, but $\left(\mathcal{D}_{\mu} X\right)_{1,3}=D_{\mu} X_{1,3}+\left[K_{\mu}, X_{3,1}\right]$.

Plugging the expansion (2.10) into the action (2.4) and using the identities

$$
\begin{aligned}
\varepsilon^{\mu \nu} D_{\mu} D_{\nu} & =-\varepsilon^{\mu \nu} \text { ad } K_{\mu} \text { ad } K_{\nu} \\
\varepsilon^{\mu \nu}\left[D_{\mu}, \text { ad } K_{\nu}\right] & =0 \\
{\left[D_{\mu}, \sqrt{h} h^{\mu \nu} \text { ad } K_{\nu}\right] } & =0
\end{aligned}
$$

which follow from the equations of motion (2.6), one can bring the quadratic part of the action to the form

$$
\begin{aligned}
S= & \bar{S}+\int d^{2} x \sqrt{h} h^{\mu \nu} \operatorname{Str}\left(\frac{1}{2} D_{\mu} X_{2} D_{\nu} X_{2}-\frac{1}{2}\left[K_{\mu}, X_{2}\right]\left[K_{\nu}, X_{2}\right]\right. \\
& \left.+X_{1} \nabla_{+\mu}\left[K_{-\nu}, X_{1}\right]+X_{3} \nabla_{-\mu}\left[K_{+\nu}, X_{3}\right]-2\left[K_{+\mu}, X_{3}\right]\left[K_{-\nu}, X_{1}\right]\right) \\
& +O\left(\kappa X^{3}\right),
\end{aligned}
$$

where the chiral projections of a vector are defined in (2.8). In the conformal gauge $\left(h_{\mu \nu}=\mathrm{e}^{\phi} \delta_{\mu \nu}\right)$, the quadratic part of the Lagrangian becomes:

$$
\begin{aligned}
\mathcal{L}_{2}=\frac{1}{2} \operatorname{Str} & \left(\bar{D} X_{2} D X_{2}-\left[\bar{K}, X_{2}\right]\left[K, X_{2}\right]\right. \\
& \left.+X_{1} D\left[\bar{K}, X_{1}\right]+X_{3} \bar{D}\left[K, X_{3}\right]-2\left[K, X_{3}\right]\left[\bar{K}, X_{1}\right]\right)
\end{aligned}
$$

where holomorphic and anti-holomorphic vector components are defined as $D=D_{1}+i D_{2}$, $\bar{D}=D_{1}-i D_{2}$, and similarly for $K$.

The fermion fluctuations of the worldsheet couple to the background currents, and if the currents vanish the fermion kinetic terms vanish too. Even if the background currents do not vanish, the Dirac operator may have zero modes, because the Lagrangian depends on $X_{1}$ $\left(X_{3}\right)$ only in the combination $\left[\bar{K}, X_{1}\right]\left(\left[K, X_{3}\right]\right)$. If $\bar{K}(K)$ has a non-empty commutant in $\mathfrak{h}_{1}$ 
$\left(\mathfrak{h}_{3}\right)$, the Lagrangian degenerates and simply does not depend on the fermionic fluctuations in the corresponding directions. This is a manifestation of the $\kappa$-symmetry, a local fermion gauge invariance that has to be fixed in order to have well-defined perturbation theory.

The most simple and natural way to fix the kappa-gauge is to set to zero those components of $X_{1}$ and $X_{3}$ that drop out from the action anyway. These components are proportional to the Lie algebra generators from $\mathfrak{h}_{1}$ and $\mathfrak{h}_{3}$ which are annihilated by the adjoint action of $\bar{K}$ or $K$. The rank of the $\kappa$-symmetry is the number of such generators:

$$
N_{\kappa}=\left.\operatorname{dim} \operatorname{ker} \operatorname{ad} K\right|_{\mathfrak{h}_{3}}, \quad N_{\tilde{\kappa}}=\left.\operatorname{dim} \operatorname{ker} \operatorname{ad} \bar{K}\right|_{\mathfrak{h}_{1}},
$$

where $K$ and $\bar{K}$ are sufficiently generic null elements of $\mathfrak{h}_{2}$. The null condition follows from the Virasoro constraints

$$
\operatorname{Str} K^{2}=0=\operatorname{Str} \bar{K}^{2} .
$$

The number of zero modes $N_{\kappa}$ or $N_{\tilde{\kappa}}$ does not depend on the particular choice of $K$ and $\bar{K}$ provided that they are sufficiently generic. For special (non-generic) classical solutions, the kappa-symmetry gauge condition may further degenerate. This is known to happen in $A d S_{4} \times C P^{3}[18]$. However these degenerate cases occur on the surface of non-vanishing co-dimension in phase space. In the bulk of the phase space (for generic classical solutions) the rank of the kappa-symmetry is background independent, and is determined by the structure constants of the Lie superalgebra $\mathfrak{g}$.

\section{Beta function and central charge}

To compute the central charge and the beta-function of the sigma-model, we integrate out $X_{n}, n=1,2,3$ in (2.12) and study the dependence of the effective action on the background currents and the $2 \mathrm{~d}$ metric. The beta-function is determined by the log-divergent contribution to the unique dimension two operator: $\sqrt{h} h^{\mu \nu} \operatorname{Str} K_{\mu} K_{\nu}$. The central charge is determined by the standard conformal anomaly. Since the beta function and the central charge are governed by different terms in the effective action, they can be computed separately. The beta function arises from the insertions of the mass operators $K^{2} X_{2}^{2}$ and $K^{2} X_{1} X_{3}$ in the one-loop diagram and can be calculated in the conformal gauge. The central charge arises due to the short-distance anomaly in the fluctuation determinants and is insensitive to the masses. In computing the central charge the masses can thus be omitted, after which the Lagrangian (2.12) reduces to that of the Green-Schwarz string in flat space in the semi-light-cone gauge, the central charge for which was computed in [25-27].

The one-loop effective action in the conformal gauge is

$$
S_{\text {eff }}=\frac{1}{2} \operatorname{Sp}_{2} \ln (-\bar{D} D+\operatorname{ad} K \operatorname{ad} \bar{K})-\frac{1}{2} \operatorname{Sp}_{1 \oplus 3}^{\prime}\left(\begin{array}{cc}
\operatorname{ad} K \operatorname{ad} \bar{K} & \bar{D} \operatorname{ad} K \\
D \operatorname{ad} \bar{K} & \operatorname{ad} \bar{K} \operatorname{ad} K
\end{array}\right) .
$$

Here we used that $-D^{\mu} D_{\mu}+$ ad $K^{\mu}$ ad $K_{\mu}=-\bar{D} D+\operatorname{ad} K$ ad $\bar{K}$ because of the identity $\left[D_{\mu}, D_{\nu}\right]=\operatorname{ad} F_{\mu \nu}=-\left[\operatorname{ad} K_{\mu}\right.$, ad $\left.K_{\nu}\right]$ satisfied by the background currents in virtue of the equations of motion (2.6). The prime in $\mathrm{Sp}_{1 \oplus 3}^{\prime}$ means that the zero eigenvectors of ad $\bar{K}$ 


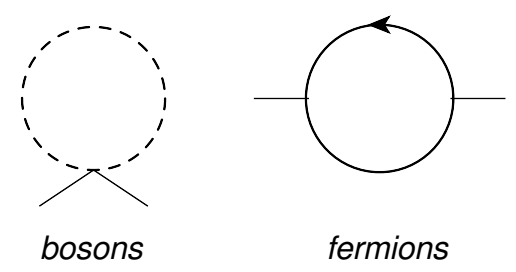

Figure 1. The one-loop contribution to the beta-function.

$(\operatorname{ad} K)$ in $\mathfrak{h}_{1}\left(\mathfrak{h}_{3}\right)$ should be omitted. They are eliminated by fixing the kappa-symmetry gauge.

The log-divergent contribution to the beta-function comes from the two diagrams in figure 1. The bosonic contribution is easy to compute:

$$
\frac{1}{2} \int \frac{d^{2} p}{(2 \pi)^{2}} \frac{1}{p^{2}} \int d^{2} x \operatorname{tr}_{2}\left(\operatorname{ad} K_{\mu}\right)^{2}=\frac{1}{4 \pi} \ln \Lambda \int d^{2} x \operatorname{tr}_{2}\left(\operatorname{ad} K_{\mu}\right)^{2} .
$$

The fermion contribution requires more care because of the kappa-symmetry projection. The Dirac operator in (3.1) can be factorized as

$$
\begin{aligned}
\left(\begin{array}{cc}
\operatorname{ad} K \operatorname{ad} \bar{K} & \bar{D} \operatorname{ad} K \\
D \operatorname{ad} \bar{K} & \operatorname{ad} \bar{K} \operatorname{ad} K
\end{array}\right) & =\left(\begin{array}{cc}
\bar{D} & \operatorname{ad} K \\
\operatorname{ad} \bar{K} & D
\end{array}\right)\left(\begin{array}{cc}
0 & \operatorname{ad} K \\
\operatorname{ad} \bar{K} & 0
\end{array}\right) \\
& =\left(\begin{array}{cc}
0 & \operatorname{ad} K \\
\operatorname{ad} \bar{K} & 0
\end{array}\right)\left(\begin{array}{cc}
D & \operatorname{ad} K \\
\operatorname{ad} \bar{K} & \bar{D}
\end{array}\right)
\end{aligned}
$$

where we used that $[D$, ad $\bar{K}]=0=[\bar{D}$, ad $K]$ due to the equations of motion. The Dirac operator acts on $\mathfrak{h}_{1} \oplus \mathfrak{h}_{3}$, so the factor

$$
\left(\begin{array}{cc}
0 & \operatorname{ad} K \\
\operatorname{ad} \bar{K} & 0
\end{array}\right)
$$

is just the kappa-symmetry projector, up to proportionality factor. The fermion contribution to the effective action thus is given by

$$
S_{\text {eff }}^{(\text {ferm })}=-\frac{1}{2} \operatorname{Sp}_{1 \oplus 3}^{\prime}\left(\begin{array}{cc}
\bar{D} & \operatorname{ad} K \\
\operatorname{ad} \bar{K} & D
\end{array}\right) .
$$

Expanding to the second order in ad $K$, ad $\bar{K}$, we find:

$$
\begin{aligned}
\frac{1}{4} \int \frac{d^{2} p}{(2 \pi)^{2}} & \frac{1}{p^{2}} \int d^{2} x\left(\operatorname{tr}_{1}^{\prime} \operatorname{ad} K \operatorname{ad} \bar{K}+\operatorname{tr}_{3}^{\prime} \operatorname{ad} \bar{K} \operatorname{ad} K\right) \\
= & \frac{1}{8 \pi} \ln \Lambda \int d^{2} x\left(\operatorname{tr}_{1} \operatorname{ad} K \operatorname{ad} \bar{K}+\operatorname{tr}_{3} \operatorname{ad} \bar{K} \operatorname{ad} K\right)
\end{aligned}
$$

The prime in the trace is omitted in the second line because the integrand is proportional to the kappa-symmetry projector. Now,

$$
\begin{aligned}
& \operatorname{ad} K \operatorname{ad} \bar{K}=\operatorname{ad} K_{\mu} \operatorname{ad} K^{\mu}-i \varepsilon^{\mu \nu} \operatorname{ad} K_{\mu} \operatorname{ad} K_{\nu} \\
& \operatorname{ad} \bar{K} \operatorname{ad} K=\operatorname{ad} K_{\mu} \operatorname{ad} K^{\mu}+i \varepsilon^{\mu \nu} \operatorname{ad} K_{\mu} \operatorname{ad} K_{\nu},
\end{aligned}
$$


so it might seem that fermions renormalize also the operator $\Sigma_{a b} \varepsilon^{\mu \nu} K_{\mu}^{a} K_{\nu}^{b}$, where $\Sigma_{a b}$ is an anti-symmetric invariant tensor on $\mathfrak{h}_{2}$. However, this operator is a total derivative, its variation being proportional to $\varepsilon^{\mu \nu} D_{\mu} K_{\nu}=0$, and integrates to zero.

Adding together bosonic and fermionic contributions we find:

$$
S_{\text {eff }}=\frac{1}{8 \pi} \ln \Lambda \int d^{2} x\left(2 \operatorname{tr}_{2}-\operatorname{tr}_{1}-\operatorname{tr}_{3}\right)\left(\operatorname{ad} K_{\mu}\right)^{2}+\text { finite. }
$$

Finally, recalling that $K_{\mu} \in \mathfrak{h}_{2}$ and thus ad $K_{\mu}$ maps $\mathfrak{h}_{2}$ to $\mathfrak{h}_{0}$ and vice versa, we find:

$$
\operatorname{tr}_{2} \operatorname{ad} K_{\mu} \operatorname{ad} K^{\mu}=\operatorname{tr}_{0} \operatorname{ad} K^{\mu} \text { ad } K_{\mu} .
$$

Hence we can replace $2 \operatorname{tr}_{2}-\operatorname{tr}_{1}-\operatorname{tr}_{3}$ in (3.4) by $\operatorname{tr}_{0}+\operatorname{tr}_{2}-\operatorname{tr}_{1}-\operatorname{tr}_{3}=\operatorname{Str}_{\text {adj }}$.

If we denote the Hermitian generators of $\mathfrak{h}_{2}$ by $T_{a}$ and introduce the metric on the bosonic section of the coset:

$$
g_{a b}=\frac{1}{\kappa^{2}} \operatorname{Str} T_{a} T_{b}
$$

the one loop beta-function is

$$
\beta_{a b}^{1-\text { loop }}=\frac{d}{d \ln \Lambda} g_{a b}=-\frac{1}{4 \pi} f_{a B}^{A} f_{b A}^{B}(-1)^{|A|},
$$

where $f_{B C}^{A}$ are the structure constants of $\mathfrak{g}$. The beta-function is thus proportional to the Killing form. The same one-loop beta-function arises in the pure-spinor-type cosets $[2,9$, $22]$, the supergroup principal field [28, 29], and in the $\mathbb{Z}_{2}$ cosets of supergroups [30, 31]. The condition for the one-loop beta-function to vanish is that the Killing form of $\mathfrak{g}$ vanishes. ${ }^{6}$

The calculation of the central charge for the Green-Schwarz string requires careful regularization of the integration measure [34-37], and yields the following result [8, 25-27]: the bosons have central charge 1; the left (right) moving fermions contribute 2 to the left (right) central charge. In our case, $X_{3}$ and $X_{1}$ are, respectively, left and right movers so, in total,

$$
c_{L}=\operatorname{dim} \mathfrak{h}_{2}+2\left(\operatorname{dim} \mathfrak{h}_{3}-N_{\kappa}\right), \quad c_{R}=\operatorname{dim} \mathfrak{h}_{2}+2\left(\operatorname{dim} \mathfrak{h}_{1}-N_{\tilde{\kappa}}\right) .
$$

The average central charge, $c=\left(c_{L}+c_{R}\right) / 2$, is determined by the dimension of the coset and the full rank of the kappa-symmetry:

$$
c=\operatorname{dim} G / H_{0}-N_{\kappa}-N_{\tilde{\kappa}}
$$

The central charge is manifestly positive, in contradistinction to the non-unitary $\mathbb{Z}_{2}$ supercosets, which can have negative central charge [31]. ${ }^{7}$ By an explicit calculation we will find that in all conformal $\operatorname{cosets} c_{L}=c_{R}$. We can thus make no distinction between $c, c_{L}$ and $c_{R}$.

We will be also interested in the case when an external CFT is added to the coset. At first sight, this cannot change the central charge counting, because the coset and the external CFT interact only via $2 \mathrm{~d}$ metric which does not carry dynamical degrees of freedom and

\footnotetext{
${ }^{6}$ Strictly speaking, only the projection of the Killing form on $\mathfrak{h}_{2}$ should vanish, but if the Killing form is non-vanishing it is also non-degenerate and unique [32, 33], and consequently proportional to the tree-level action thus giving a non-zero beta-function.

${ }^{7}$ Typically, the central charge of a $\mathbb{Z}_{2}$ coset is equal to its superdimension [38] which counts bosons and fermions with opposite signs.
} 
can be eliminated by fixing the conformal gauge. However, this is not quite true. Adding an external CFT can partially or completely break the kappa-symmetry. The kappa-symmetry transformations act on the $2 \mathrm{~d}$ metric and since the latter enters the action of the external CFT, kappa-symmetry gets broken. In the conformal gauge, the kappa-symmetry breaking can be attributed to the violation of the null condition for the currents (2.15), which does not hold in the presence of another CFT with a non-trivial energy-momentum tensor. ${ }^{8}$

The ranks of the left- and right-moving kappa-symmetries with the null condition relaxed will be denoted by $\hat{N}_{\kappa}, \hat{N}_{\tilde{\kappa}}$. They are computed by the same formulas (2.14) where $K$ and $\bar{K}$ are now the most general elements of $\mathfrak{h}_{2}$, not necessarily null. We will denote the central charge of the sigma-model coupled to an external CFT (the extrinsic central charge) by ${ }^{9} \hat{c}$ :

$$
\hat{c}=\operatorname{dim} G / H_{0}-\hat{N}_{\kappa}-\hat{N}_{\tilde{\kappa}}
$$

In the next section we will compute extrinsic and intrinsic central charges for all conformal $\mathbb{Z}_{4}$ cosets.

\section{Conformal sigma models}

The string sigma-model must be defined on a real superspace, so the symmetry algebra $\mathfrak{g}$ should be a real Lie superalgebra. However, the one-loop beta-function and the central charge depend only on the structure constants of $\mathfrak{g}$ and therefore are the same for all real forms of a given complex superalgebra. Dealing with complex Lie superalgebras is technically simpler, and subsequent analysis will be done as if $\mathfrak{g}$ were complex. We will pick a particular real form in the very end. If we want to have a string interpretation of the sigma-model, the real form must be such that the metric (3.5) has the Minkowski signature $(-+\cdots+)$. In the cases when the requisite real form does not exist, we will keep in mind the compact form of the coset with the $(+\cdots+)$ metric.

The basic complex Lie superalgebras with vanishing Killing form form two infinite series: $\mathfrak{p s u}(n \mid n)$ and $\mathfrak{o s p}(2 n+2 \mid 2 n)$ [32,33]. The one-parameter family of exceptional superalgebras $\mathfrak{d}(2,1 ; \alpha)$, a continuous deformation of $\mathfrak{o} \mathfrak{s p}(4 \mid 2)$, also has vanishing Killing form. But since the deformation parameter appears only in the anti-commutator of supercharges, the central charge counting for $\mathfrak{d}(2,1 ; \alpha)$ is the same as for $\mathfrak{o s p}(4 \mid 2)$ and we need not discuss $\mathfrak{d}(2,1 ; \alpha)$ separately, just keeping in mind that any $\operatorname{OSp}(4 \mid 2)$ coset can be generalized to $D(2,1 ; \alpha)$.

From the discussion above we see that there are two series of conformal sigma-models on semi-symmetric superspaces, those with $\operatorname{PSU}(n \mid n)$ and $\operatorname{OSp}(2 n+2 \mid 2 n)$ symmetry, which we will call type- $U$ and type- $O$ models. All possible $\mathbb{Z}_{4}$ automorphisms of $\mathfrak{p s u}(n \mid n)$ and $\mathfrak{o s p}(2 n+2 \mid 2 n)$ and the corresponding cosets were classified by Serganova [1]. They fall into six separate classes, four type- $U$ and two type- $O$, conveniently described with the help of

\footnotetext{
${ }^{8}$ The observation that coupling to an external CFT breaks kappa-symmetry was made in [20], but perhaps this simple fact was known before.

${ }^{9}$ This should not be confused with $2 c / 3$ sometimes also denoted by $\hat{c}$.
} 


\begin{tabular}{|c|c|c|}
\hline Coset & $\boldsymbol{\Omega}$ & $\mathbf{H}_{\mathbf{0}}$ \\
\hline type- $U 1$ & $\operatorname{Ad} \operatorname{diag}\left(I_{p}, I_{q}\right) \circ \delta$ & $\mathrm{U}(p) \times \mathrm{SU}(n-p) \times \mathrm{U}(q) \times \mathrm{SU}(n-q)$ \\
\hline type- $U 2$ & $-s t$ & $\mathrm{SO}(n) \times \mathrm{SO}(n)$ \\
\hline type- $U 3$ & $-s t \circ \Pi \circ \delta$ & $\mathrm{SU}(n)$ \\
\hline type- $U 4$ & $-s t \circ \operatorname{Ad} \operatorname{diag}(J, J)$ & $\mathrm{Sp}(n) \times \mathrm{Sp}(n)$ \\
\hline
\end{tabular}

Table 1. Semi-symmetric cosets of $\operatorname{PSU}(n \mid n) . \Omega$ is the $\mathbb{Z}_{4}$ automorphism of $\mathfrak{s u}(n \mid n), H_{0}$ is the invariant subgroup. The bosonic section of the coset is $\mathrm{SU}(n) \times \mathrm{SU}(n) / H_{0}$.

\begin{tabular}{|c|c|c|}
\hline Coset & $\boldsymbol{\Omega}$ & $\mathbf{H}_{\mathbf{0}}$ \\
\hline type-O1 & $\operatorname{Ad} \operatorname{diag}\left(I_{p}, J\right)$ & $\mathrm{SO}(p) \times \mathrm{SO}(2 n+2-p) \times \mathrm{U}(n)$ \\
\hline type-O2 & $\operatorname{Ad} \operatorname{diag}\left(J, \mathbb{1} \otimes I_{p}\right)$ & $\mathrm{U}(n+1) \times \mathrm{Sp}(2 p) \times \mathrm{Sp}(2 n-2 p)$ \\
\hline
\end{tabular}

Table 2. Semi-symmetric cosets of $\operatorname{OSp}(2 n+2 \mid 2 n) . \Omega$ is the $\mathbb{Z}_{4}$ automorphism of $\mathfrak{o s p}(2 n+2 \mid 2 n)$, $H_{0}$ is the invariant subgroup. The bosonic section of the coset is $\mathrm{SO}(2 n+2) \times \mathrm{Sp}(2 n) / H_{0}$.

the supermatrix representation of the $\mathfrak{s u}(n \mid n)$ and $\mathfrak{o s p}(2 n+2 \mid 2 n)$ superalgebras: ${ }^{10}$

$$
\begin{aligned}
\mathfrak{s u}(n \mid n) & =\{X \in M(n \mid n) \mid \operatorname{Str} X=0\} \\
\mathfrak{o s p}(2 n+2 \mid 2 n) & =\left\{\left(\begin{array}{ll}
A & \Theta \\
\Psi & B
\end{array}\right) \in M(2 n+2 \mid 2 n) \mid A=-A^{t}, B=J B^{t} J, \Psi=J \Theta^{t}\right\} .
\end{aligned}
$$

Here $J$ is the $2 n \times 2 n$ matrix

$$
J=\left(\begin{array}{cc}
0 & \mathbb{1}_{n \times n} \\
-\mathbb{1}_{n \times n} & 0
\end{array}\right) .
$$

We will also need the diagonal matrix

$$
I_{p}=\operatorname{diag}\left(\mathbb{1}_{p \times p},-\mathbb{1}_{(n-p) \times(n-p)}\right),
$$

and the following supermatrix operations: ${ }^{11}$

$$
\begin{aligned}
\left(\begin{array}{ll}
A & \Theta \\
\Psi & B
\end{array}\right)^{s t} & =\left(\begin{array}{cc}
A^{t} & -\Psi^{t} \\
\Theta^{t} & B^{t}
\end{array}\right) \\
\delta \circ\left(\begin{array}{ll}
A & \Theta \\
\Psi & B
\end{array}\right) & =\left(\begin{array}{cc}
A & i \Theta \\
-i \Psi & B
\end{array}\right) \\
\Pi \circ\left(\begin{array}{ll}
A & \Theta \\
\Psi & B
\end{array}\right) & =\left(\begin{array}{cc}
B & \Psi \\
\Theta & A
\end{array}\right)
\end{aligned}
$$

These three operations and the adjoint action of the matrices $J$ and $I_{p}$ allow one to build all possible $\mathbb{Z}_{4}$ automorphisms of $\mathfrak{s u}(n \mid n)$ (table 1 ) and $\mathfrak{o s p}\left(2 n+2 \mid 2 n\right.$ ) (table 2) [1]. The $\mathbb{Z}_{4}$ decomposition corresponding to these cosets is described in more detail in the appendix.

Almost all of the cosets in tables 1 and 2 can be used to define the action of a sigmamodel, except for type- $U 3$. Any element of $\mathfrak{h}_{2}$ in a type- $U 3$ coset is null, at least in the

\footnotetext{
${ }^{10}$ The central element in $\mathfrak{s u}(n \mid n)$ that distinguishes it from $\mathfrak{p s u}(n \mid n)$ can be trivially factored out.

${ }^{11}$ Our notations are essentially identical to those of [1].
} 
usual supertrace metric (the explicit $\mathbb{Z}_{4}$ decomposition is given in section A.3). The bosonic part of the action then vanishes identically. Although we will formally compute the central charge for this coset, we will not discuss this sigma-model any further.

In addition to the models based on simple Lie superalgebras one can also consider the cosets of product groups. Such cosets naturally arise in the $A d S_{3} / C F T_{2}$ correspondence, because the conformal algebra in two dimensions is a direct sum of two Virasoro algebras acting independently on the left and right movers. Independently of the AdS/CFT connection, the product structure is quite natural from the point of view of the coset construction, as it generally admits a $\mathbb{Z}_{4}$ action. If $\mathfrak{p}$ is a superalgebra, we can define a $\mathbb{Z}_{4}$ action on the direct sum $\mathfrak{g}=\mathfrak{p} \oplus \mathfrak{p}$ by combining the permutation of the two factors with the fermion number [20]:

$$
\Omega=\left(\begin{array}{cc}
0 & \text { id } \\
(-1)^{F} & 0
\end{array}\right) .
$$

One can easily check that $\Omega([X, Y])=[\Omega(X), \Omega(Y)]$ for any $X, Y \in \mathfrak{p} \oplus \mathfrak{p}$. It is also obvious that $\Omega^{2}=(-1)^{F}$ and thus $\Omega^{4}=\mathrm{id}$. The invariant subalgebra of the $\mathbb{Z}_{4}$ action is the bosonic diagonal $\mathfrak{h}_{0}=\{(X, X) \mid X \in \mathfrak{p}\}$. Consequently, the supercoset is $P \times P / H_{0}$, where $H_{0}$ is the bosonic subgroup of $P$ diagonally embedded in $P \times P$. The bosonic section is the group manifold of $H_{0}$. We refer to the tensor-product semi-symmetric spaces as type- $T u$ cosets if $P=\operatorname{PSU}(n \mid n)$ and type-To cosets if $P=\operatorname{OSp}(2 n+2 \mid 2 n)$. There are also interesting cosets of $\mathrm{U}(n \mid n)[39]$, which we will not consider here.

We will calculate the one-loop central charge for the eight types of semi-symmetric cosets introduced above (type- $U 1-4$, type-O1,2 and type- $T u, o$ ). The central charge counts the number of degrees of freedom in the sigma-model and depends on the rank of the kappa-symmetry (3.7)-(3.9), which in turn is given by the dimension of the commutant of a generic element of $\mathfrak{h}_{2}$ (2.14). The calculations reduce to simple algebra, but have to be done case by case. The details are given in the appendix, here we just describe the general pattern that emerges:

- The left- and right-moving kappa-symmetries, which are associated with the $\mathfrak{h}_{3}$ and $\mathfrak{h}_{1}$ subspaces, are identical in almost all the cases. One and only exception is the type- $U 3$ coset, for which $\mathfrak{h}_{3}$ and $\mathfrak{h}_{1}$ are not isomorphic and have different dimensions. The kappa-symmetry compensates for this, such that even in this case the left- and right-moving central charges are equal.

- The extrinsic kappa-symmetries follow a regular pattern and depend uniformly on the dimensionalities of the superalgebra and the coset (table 3). ${ }^{12}$

- There is no difference between intrinsic and extrinsic kappa-symmetries and central charges in most cases, but in low ranks there are exceptions listed in table 4. Imposing the Virasoro constraints then increases the rank of the kappa-symmetry and decreases the central charge.

\footnotetext{
${ }^{12}$ For type- $U 1$, we assume that $n \geqslant 2 p$ and $n \geqslant 2 q$.
} 


\begin{tabular}{|c|c|c|}
\hline Coset & $\hat{\mathbf{N}}_{\kappa}$ & $\hat{\mathbf{N}}_{\tilde{\kappa}}$ \\
\hline type- $U 1$ & $(n-2 p)(n-2 q)$ & $(n-2 p)(n-2 q)$ \\
\hline type- $U 2$ & 0 & 0 \\
\hline type- $U 3$ & 0 & $2 n$ \\
\hline type- $U 4$ & 0 & 0 \\
\hline type- $O 1$ & 0 & 0 \\
\hline type- $O 2(n$ odd $)$ & 0 & 0 \\
\hline type-O2 $(n$ even $)$ & $2 n-4 p$ & $2 n-4 p$ \\
\hline type- $T u$ & 0 & 0 \\
\hline type- $T o$ & 0 & 0 \\
\hline
\end{tabular}

Table 3. The rank of the kappa-symmmetry, generic case.

\begin{tabular}{|c|c|}
\hline Coset & $\mathbf{N}_{\kappa}$ \\
\hline type- $U 1(p=1, q=1)$ & $n^{2}-4 n+6$ \\
\hline type- $U 2(n=2)$ & 2 \\
\hline type- $U 4(n=4)$ & 8 \\
\hline type- $O 1(n=1, p=1)$ & 1 \\
\hline type- $O 2(n=2, p=1)$ & 4 \\
\hline type- $T u(n=2)$ & 4 \\
\hline
\end{tabular}

Table 4. The rank of the kappa-symmetry, exceptions (in all cases $N_{\tilde{\kappa}}=N_{\kappa}$ ).

\begin{tabular}{|c|c|}
\hline Coset & $\hat{\mathbf{c}}$ \\
\hline type- $U 1$ & $6(p+q) n-2 p^{2}-2 q^{2}-8 p q$ \\
\hline type- $U 2$ & $3 n^{2}+n-2$ \\
\hline type- $U 3$ & $3 n^{2}-2 n-1$ \\
\hline type- $U 4$ & $3 n^{2}-n-2$ \\
\hline type- $O 1$ & $5 n^{2}+(2 p+5) n-p^{2}+2 p$ \\
\hline type- $O 2(n$ odd $)$ & $5 n^{2}+(4 p+5) n-4 p^{2}$ \\
\hline type- $O 2(n$ even $)$ & $5 n^{2}+(4 p+1) n-4 p^{2}+8 p$ \\
\hline type- $T u$ & $6 n^{2}-2$ \\
\hline type- $T o$ & $12 n^{2}+12 n+1$ \\
\hline
\end{tabular}

Table 5. The central charge, regular case.

The central charges for the regular cosets (for which there is no difference between $\hat{c}$ and c) are summarized in table 5. The exceptional cases in which the intrinsic and extrinsic central charges are different are listed in table 6 .

\section{$5 \quad$ String sigma-models}

The worldsheet diffeomorphisms in the sigma-models at hand are just the same as in the bosonic string theory, and lead to the same set of $b c$ ghosts in the conformal gauge. If the 


\begin{tabular}{|c|c|c|}
\hline Coset & c & $\hat{\mathbf{c}}$ \\
\hline type- $U 1(p=1, q=1)$ & $12 n-16$ & $12 n-12$ \\
\hline type- $U 2(n=2)$ & 8 & 12 \\
\hline type- $U 4(n=4)$ & 26 & 42 \\
\hline type- $O 1(n=1, p=1)$ & 11 & 13 \\
\hline type- $O 2(n=2, p=1)$ & 26 & 34 \\
\hline type- $T u(n=2)$ & 14 & 22 \\
\hline
\end{tabular}

Table 6. The central charge, exceptions.

ghost contribution to the central charge is to be canceled by the sigma-model alone, its intrinsic central charge should be equal to 26 . If $\hat{c}<26$, the central charge deficit can be compensated by coupling to an external CFT with central charge $26-\hat{c}$. Let us list the models that satisfy these criteria.

The inspection of tables 5 and 6 shows that there are only two cosets with $c=26$, both are exceptional:

$$
\begin{array}{lrr}
\text { Type- } U 4(n=4): \quad & \mathrm{PU}(2,2 \mid 4) / \mathrm{SO}(4,1) \times \mathrm{SO}(5) & A d S_{5} \times S^{5} \\
\text { Type-O2(n=2,p=1) }: \quad \operatorname{OSp}(6 \mid 4) / \mathrm{U}(3) \times \mathrm{SO}(3,1) & A d S_{4} \times C P^{3}
\end{array}
$$

These cosets define well-known sigma-models. The first one is the Metsaev-Tseytlin model for the Green-Schwarz superstring on $A d S_{5} \times S^{5}$ [3]. The second model describes strings on $A d S_{3} \times C P^{3}[18,19]$ and can be obtained from the Green-Schwarz action on this background [40] by partially fixing kappa-symmetry. It is interesting that these two cases are in a sense unique.

There is a number of non-critical semi-symmetric cosets with $\hat{c}<26$. Some of them admit a real form with the Minkowski metric on the bosonic subspace:

1) Type-To(n=1): $\quad \operatorname{OSp}(4 \mid 2) \times \mathrm{OSp}(4 \mid 2) / \mathrm{SO}(4) \times \mathrm{SL}(2, \mathbb{R})$

$$
\hat{c}=25 \quad A d S_{3} \times S^{3} \times S^{3}
$$

2) Type $-T u(n=2): \quad \operatorname{PSU}(1,1 \mid 2) \times \operatorname{PSU}(1,1 \mid 2) / \mathrm{SU}(1,1) \times \mathrm{SU}(2)$

$$
\hat{c}=22 \quad A d S_{3} \times S^{3}
$$

3) Type-O1 $(n=1, p=2): \quad \operatorname{OSp}(4 \mid 2) / \mathrm{U}(1)^{3}$

$$
\hat{c}=14 \quad A d S_{2} \times S^{2} \times S^{2}
$$

4) Type-O1 $(n=1, p=1): \quad \operatorname{OSp}(4 \mid 2) / \mathrm{SO}(3) \times \mathrm{U}(1)$

$$
\hat{c}=13 \quad A d S_{2} \times S^{3}
$$

5) Type- $U 1(n=2, p=1, q=1) / U 2(n=2): \quad \operatorname{PSU}(1,1 \mid 2) / \mathrm{U}(1)^{2}$

$$
\hat{c}=12 \quad A d S_{2} \times S^{2}
$$

6) Type $-U 1(n=2, p=1, q=0): \quad \operatorname{PSU}(1,1 \mid 2) / \mathrm{U}(1) \times \mathrm{SU}(2)$

$$
\hat{c}=10 \quad A d S_{2}
$$

7) Type-O1 $(n=1, p=0) / O 2(n=1, p=0): \quad \operatorname{OSp}(4 \mid 2) / \mathrm{SO}(4) \times \mathrm{U}(1)$

$$
\hat{c}=10 \quad A d S_{2}
$$


Here $\operatorname{OSp}(4 \mid 2)$ can be replaced by a more general supergroup $D(2,1 ; \alpha)$, which gives a one-parametric family of sigma-models. In particular, there is a $D(2,1 ; \alpha)$ coset which continuously interpolates between cases 7 and 6 . The same is true for cases 1 and 2 [20], where the degeneration of $D(2,1 ; \alpha)$ to $\operatorname{PSU}(1,1 \mid 2)$ leaves two extra flat dimensions which account for the difference in central charges.

Many of the cosets above have been discussed in the context of the AdS/CFT duality. The first coset, supplemented by an external $S^{1}$, describes the Green-Schwarz string on $A d S_{3} \times S^{3} \times S^{3} \times S^{1}$ with completely fixed kappa-symmetry [20]. The action of the second coset can be interpreted as the $6 \mathrm{~d}$ Green-Schwarz action on $A d S_{3} \times S^{3}[9,11,12,14,16,41]$ and as such admits rank-eight kappa-symmetry (table 4). However, coupling to an external $T^{4}$, which is necessary to compensate for the central charge deficit, breaks kappa-symmetry and changes the central charge counting. This coset plus four compact bosons describes the Green-Schwarz string on ${ }^{13} A d S_{3} \times S^{3} \times T^{4}$ with fully fixed kappa-symmetry [20]. The fifth coset yields the $4 \mathrm{~d}$ Green-Schwarz action on $A d S_{2} \times S^{2}[2,9,13]$. Again, its (four-parameter) kappa-symmetry is completely broken by coupling to an external $c=14 \mathrm{CFT}$. The models $3,4,6$ and 7 are seemingly new. The last two models are similar to the $\operatorname{OSp}(1 \mid 2) / \mathrm{U}(1)$ coset considered in [15] — they have $A d S_{2}$ as the bosonic target space and no physical degrees of freedom on shell. The latter is due to kappa-symmetry. Coupling of these sigma-models to an external CFT breaks kappa-symmetry and revives their fermion degrees of freedom.

Other non-critical cosets do not admit a metric with the $(-+\cdots+)$ signature and the time direction should lie in the external CFT. Their compact versions (those with the Euclidean metric) are listed below:

$$
\begin{array}{ll}
\text { 1) Type }-U 1(n=3, p=1, q=1): & \operatorname{PSU}(1,2 \mid 3) / \mathrm{U}(2) \times \mathrm{U}(2) \\
\hat{c}=24 \quad C P^{2} \times C P^{2} & \\
\text { 2) Type- } U 1(n=4, p=1, q=0): & \operatorname{PSU}(4 \mid 4) / \mathrm{U}(3) \times \mathrm{SU}(4) \\
\hat{c}=22 \quad C P^{3} & \\
\text { 3) Type }-O 2(n=2, p=0): & \mathrm{OSp}(6 \mid 4) / \mathrm{U}(3) \times \mathrm{Sp}(4) \\
\hat{c}=22 \quad C P^{3} & \\
\text { 4) Type- }-U 1(n=3, p=1, q=0): & \operatorname{PSU}(3 \mid 3) / \mathrm{U}(2) \times \mathrm{SU}(3) \\
\hat{c}=16 \quad C P^{2} . &
\end{array}
$$

These models bear certain resemblance to the $\mathbb{C P}^{S-1 \mid S} \mathbb{Z}_{2}$ sigma models [42].

\section{Conclusions}

The list of semi-symmetric superspaces potentially consistent as string backgrounds is not very long. We should stress that we have computed the beta function and central charge only at the one loop level. There is no guarantee that higher-order corrections identically vanish, and the list of consistent string backgrounds with the $\mathbb{Z}_{4}$ symmetry may be

\footnotetext{
${ }^{13}$ This background admits a hybrid description in terms of the sigma model on the supergroup manifold $\operatorname{PSU}(1,1 \mid 2)[28]$.
} 
even shorter. It is instructive to look at what happens in the principal chiral models and $\mathbb{Z}_{2}$ cosets of supergroups. In the case of the principal chiral field, it is possible to prove finiteness to all orders in perturbation theory for the cosets with the vanishing one-loop beta-function [29, 31]. Many one-loop finite $\mathbb{Z}_{2}$ cosets are two-loop finite as well [31], but the full set of conformal $\mathbb{Z}_{2}$ cosets seems to be smaller than the set of $\mathbb{Z}_{2}$ cosets with vanishing one-loop beta-function [43].

The semi-symmetric cosets with non-zero beta-function can also be interesting for the AdS/CFT duality, if they are asymptotically free. A sigma-model with the AdS target cannot develop a mass gap because of the non-compactness. The asymptotic freedom at weak coupling then suggests that the beta-function has a non-trivial zero, which can potentially be interpreted as string theory on the AdS space of fixed radius [8]. ${ }^{14}$

The consistent Minkowski backgrounds, critical (5.1) and non-critical (5.2), all involve an AdS factor and are potentially dual to CFTs in dimensions $d \leqslant 4$. In all these cases the worldsheet sigma-model is integrable and thus potentially solvable by Bethe ansatz. For the string sigma-models on $A d S_{5} \times S^{5}$ and $A d S_{4} \times C P^{3}$ the classical algebraic curve [44-46], the worldsheet S-matrix $[47,48]$ and the asymptotic quantum Bethe equations $[49,50]$ are known. The finite-volume TBA/Y-system solution is now also available [51-56]. It would be interesting to derive a unifying Bethe-ansatz solution for a generic semi-symmetric coset.

\section{Acknowledgments}

I would like to thank I. Adam, A. Babichenko, I. Bakas, N. Berkovits, V. Kazakov, S. Leurent, L. Mazzucato, J. Minahan, V. Mitev, Y. Oz, A. Polyakov, V. Schomerus, S. Shatashvili, B. Stefanski, A. Tseytlin, J. Troost, B. Vicedo, K. Wiese and N. Wyllard for interesting discussions and useful comments. This work was supported in part by the BQR ENS, in part by the Swedish Research Council under the contract 621-2007-4177, in part by the ANF-a grant 09-02-91005, and in part by the grant for support of scientific schools NSH-3036.2008.2.

\section{A Rank of kappa symmetry}

In this appendix we compute the rank of the kappa-symmetry for all conformal $\mathbb{Z}_{4}$ cosets. According to (2.14), the rank is equal to the dimension of the commutant of a generic element $K($ or $\bar{K}) \in \mathfrak{h}_{2}$ in $\mathfrak{h}_{1}$ and $\mathfrak{h}_{3}$. In the supermatrix representation,

$$
K(\text { or } \bar{K})=\left(\begin{array}{ll}
A & 0 \\
0 & B
\end{array}\right) .
$$

Commuting this with an odd element of the superalgebra, we find:

$$
\left[\left(\begin{array}{ll}
A & 0 \\
0 & B
\end{array}\right),\left(\begin{array}{ll}
0 & \Theta \\
\Psi & 0
\end{array}\right)\right]=\left(\begin{array}{cc}
0 & A \Theta-\Theta B \\
B \Psi-\Psi A & 0
\end{array}\right) .
$$

\footnotetext{
${ }^{14}$ This argument was suggested to the author by A.M. Polyakov.
} 
The commutator vanishes if

$$
A \Theta=\Theta B, \quad B \Psi=\Psi A .
$$

The number of solutions to these equations determines the rank of the kappa-symmetry. The dimension of the solution space for generic $A$ and $B$ determines the rank of the extrinsic kappa-symmetry $\hat{N}_{\kappa}, \hat{N}_{\tilde{\kappa}}$. To compute the rank of the intrinsic kappa-symmetries $N_{\kappa}$, $N_{\tilde{\kappa}}$, one should in addition impose the Virasoro constraints (2.15). The matrices $A$ and $B$ then satisfy

$$
\operatorname{tr} A^{2}=\operatorname{tr} B^{2} .
$$

Throughout the calculation we will use a number of simple algebraic facts, which we collect below.

Consider an equation for an $m \times n$ matrix $X$ :

$$
M X=X N
$$

where $M$ and $N$ are given quadratic matrices, which we assume to be sufficiently generic. Since a generic matrix can be diagonalized by a similarity transformation, without loss of generality we can assume that $M$ and $N$ are diagonal. Denoting their eigenvalues by $\mu_{i}$, $i=1, \ldots, m$ and $\nu_{a}, a=1, \ldots, n$, we find

$$
\left(\mu_{i}-\nu_{a}\right) X_{i a}=0
$$

In the most general case of arbitrary $M$ and $N$, all $\mu_{i}$ are different from $\nu_{a}$, and consequently (A.4) has no solutions other than $X=0$. This might not be true if $M$ and $N$ satisfy extra conditions. For instance, if $M$ and $N$ are $2 \times 2$ matrices constrained by

$$
\operatorname{tr} M=0=\operatorname{tr} N, \quad \operatorname{tr} M^{2}=\operatorname{tr} N^{2},
$$

their eigenvalues coincide pairwise: $\mu_{1}=-\mu_{2}=\nu_{1}=-\nu_{2}$. In this case the equation (A.4) has two linearly independent solutions. In general the rank of the linear system (A.4) is equal to the number of pairs of coinciding eigenvalues of matrices $M$ and $N$.

In analyzing the spectrum of various matrices we will repeatedly use the Stenzel theorem $[57,58]$, which states that the non-zero eigenvalues of a product of two anti-symmetric matrices are doubly degenerate. Namely, the spectrum of an $n \times n$ matrix $M=A_{1} A_{2}$, where $A_{i}^{t}=-A_{i}$, consists of $[n / 2]$ pairs of eigenvalues $\mu_{1}, \mu_{1}, \ldots \mu_{[n / 2]}, \mu_{[n / 2]}$ and, if $n$ is odd, an additional zero eigenvalue associated with the vector annihilated by $A_{2}$.

\section{A.1 Type- $U 1$}

The $\mathbb{Z}_{4}$ automorphism of the type- $U 1$ coset acts on the supermatrices as

$$
\Omega \circ\left(\begin{array}{cc}
A & \Theta \\
\Psi & B
\end{array}\right)=\left(\begin{array}{cc}
I_{p} A I_{p} & i I_{p} \Theta I_{q} \\
-i I_{q} \Psi I_{p} & I_{q} B I_{q}
\end{array}\right),
$$


where $I_{p}, I_{q}$ are defined in (4.4). The $\mathbb{Z}_{4}$ decomposition in the supermatrix representation is given by

$$
\begin{aligned}
\mathfrak{h}_{2}: & A=\left(\begin{array}{cc}
0 & {\left[A_{1}\right]_{p \times(n-p)}} \\
{\left[A_{2}\right]_{(n-p) \times p}} & 0
\end{array}\right), & B & =\left(\begin{array}{cc}
0 & {\left[B_{1}\right]_{q \times(n-q)}} \\
{\left[B_{2}\right]_{(n-q) \times q}} & 0
\end{array}\right) \\
\mathfrak{h}_{1}: & \Theta=\left(\begin{array}{cc}
{\left[\Theta_{1}\right]_{p \times q}} & 0 \\
0 & {\left[\Theta_{2}\right]_{(n-p) \times(n-q)}}
\end{array}\right), & \Psi & =\left(\begin{array}{cc}
0 & {\left[\Psi_{1}\right]_{q \times(n-p)}} \\
{\left[\Psi_{2}\right]_{(n-q) \times p}} & 0
\end{array}\right) \\
\mathfrak{h}_{3}: & \Theta=\left(\begin{array}{cc}
0 & {\left[\Theta_{1}\right]_{p \times(n-q)}} \\
{\left[\Theta_{2}\right]_{(n-p) \times q}} & 0
\end{array}\right), & \Psi & =\left(\begin{array}{cc}
{\left[\Psi_{1}\right]_{q \times p}} & 0 \\
0 & {\left[\Psi_{2}\right]_{(n-q) \times(n-p)}}
\end{array}\right)
\end{aligned}
$$

Let us assume that $n-p \geqslant p$ and $n-q \geqslant q$. The non-zero eigenvalues of the matrix $A$ form $p$ pairs $\pm \alpha_{1}, \ldots, \pm \alpha_{p}$, where $\alpha_{i}^{2}$ are the eigenvalues of the $p \times p$ matrix $A_{1} A_{2}$. In addition $A$, has $n-2 p$ zero modes built from $(n-p)$-dimensional vectors $v_{i}$ annihilated by $A_{1}$. Analogously, the right action of $B$ produces $2 q$ non-zero eigenvalues $\pm \beta_{1}, \ldots, \pm \beta_{q}$, whose squares $\beta_{j}^{2}$ are eigenvalues of $B_{1} B_{2}$, and $n-2 q$ zero modes made of left $n-q$ dimensional null vectors of $B_{2}$, which we denote by $u_{j}$. There are $(n-2 p)(n-2 q)$ pairs of zero eigenvalues of $A$ and $B$. In $\mathfrak{h}_{1}$, they correspond to $(n-2 p)(n-2 q)$ solutions to (A.2) of the form

$$
\Psi=0, \quad \Theta_{1}=0, \quad \Theta_{2}=v_{i} \otimes u_{j}(i=1, \ldots, n-2 p ; j=1, \ldots, n-2 q) .
$$

In general, the non-zero eigenvalues $\pm \alpha_{i}$ have no reasons to coincide with $\pm \beta_{j}$, so the number of right-moving kappa-symmetries without Virasoro constraints is

$$
\hat{N}_{\tilde{\kappa}}=(n-2 p)(n-2 q) .
$$

The computation for the left-movers $\left(\mathfrak{h}_{3}\right)$ is the same with the left and right action of $A$ and $B$ interchanged and $\Theta$ replaced by $\Psi$, so

$$
\hat{N}_{\kappa}=(n-2 p)(n-2 q) .
$$

The null condition (A.3), in terms of the eigenvalues reads

$$
\sum_{i=1}^{p} \alpha_{i}^{2}=\sum_{j=1}^{q} \beta_{j}^{2} .
$$

In general, this condition is too weak and does not imply any degeneracies. The only exception is $p=1=q$, when each of the matrices $A$ and $B$ has only one pair of non-zero eigenvalues. The eq. (A.12) then implies that these eigenvalues coincide up to a sign. We thus find two extra solutions to (A.2) in both $\mathfrak{h}_{1}$ and $\mathfrak{h}_{3}$. In $\mathfrak{h}_{1}$, the solutions are

$$
\Theta_{1}=A_{1} A_{2}, \quad \Theta_{2}=A_{2} B_{1}, \quad \Psi=0
$$

and

$$
\Psi_{1}=A_{1}, \quad \Psi_{2}=B_{2}, \quad \Theta=0 .
$$

This solutions exist provided that $A_{1} A_{2}=B_{1} B_{2}$, which for $p=1=q$ is a consequence of the Virasoro constraints. Hence,

$$
N_{\kappa}=N_{\tilde{\kappa}}=(n-2)^{2}+2 \quad(p=1=q) .
$$




\section{A.2 Type- $U 2$}

The $\mathbb{Z}_{4}$ automorphism of the type- $U 2$ coset is

$$
\Omega \circ\left(\begin{array}{cc}
A & \Theta \\
\Psi & B
\end{array}\right)=\left(\begin{array}{cc}
-A^{t} & \Psi^{t} \\
-\Theta^{t} & -B^{t}
\end{array}\right),
$$

which gives the following $\mathbb{Z}_{4}$ decomposition:

$$
\begin{array}{ll}
\mathfrak{h}_{2}: & A^{t}=A, \quad B^{t}=B \\
\mathfrak{h}_{1}: & \Psi=i \Theta^{t} \\
\mathfrak{h}_{3}: & \Psi=-i \Theta^{t} .
\end{array}
$$

To find the rank of the kappa-symmetry, we need to solve the equation

$$
A \Theta=\Theta B
$$

for generic symmetric traceless matrices $A$ and $B$. It general it has no solutions, so

$$
\hat{N}_{\kappa}=0=\hat{N}_{\tilde{\kappa}} .
$$

If we impose the Virasoro condition (A.3), we are in the situation described around eq. (A.6). The exceptional case is $n=2$, in which the space of solutions to (A.18) is two-dimensional. We thus find:

$$
N_{\kappa}=N_{\tilde{\kappa}}=2 \quad(n=2) .
$$

\section{A.3 Type- $U 3$}

The $\mathbb{Z}_{4}$ automorphism in this case is

$$
\Omega \circ\left(\begin{array}{ll}
A & \Theta \\
\Psi & B
\end{array}\right)=\left(\begin{array}{cc}
-B^{t} & i \Theta^{t} \\
i \Psi^{t} & -A^{t}
\end{array}\right),
$$

which gives the following $\mathbb{Z}_{4}$ decomposition:

$$
\begin{aligned}
& \mathfrak{h}_{2}: B=A^{t} \\
& \mathfrak{h}_{1}: \Theta^{t}=\Theta, \quad \Psi^{t}=\Psi \\
& \mathfrak{h}_{3}: \Theta^{t}=-\Theta, \quad \Psi^{t}=-\Psi \text {. }
\end{aligned}
$$

This case is rather special, because $\mathfrak{h}_{1}$ and $\mathfrak{h}_{3}$ have different dimensions: $\operatorname{dim} \mathfrak{h}_{1}-\operatorname{dim} \mathfrak{h}_{3}=$ $2 n$, potentially leading to the mismatch of the central charges of left and right movers. We will see that this mismatch is precisely compensated by the kappa-symmetry.

The equation (A.2), that determines the rank of the kappa-symmetry, becomes:

$$
A \Theta=\Theta A^{t}, \quad A^{t} \Psi=\Psi A .
$$


The solutions of this equation in symmetric matrices give $\hat{N}_{\tilde{\kappa}}$, the number of solutions in anti-symmetric matrices determines $\hat{N}_{\kappa}$. Without loss of generality we can assume that $A$ is diagonal: $A=\operatorname{diag}\left(a_{1}, \ldots, a_{n}\right)$. Then,

$$
\left(a_{i}-a_{j}\right) \Theta_{i j}=0, \quad\left(a_{i}-a_{j}\right) \Psi_{i j}=0 .
$$

In general, all the eigenvalues are different, and the solutions are diagonal matrix elements $\Theta_{i i}$ and $\Psi_{i i}$. All of them belong to $\mathfrak{h}_{1}$, thus giving:

$$
\hat{N}_{\tilde{\kappa}}=2 n, \quad \hat{N}_{\kappa}=0 .
$$

The kappa-symmetry eliminates the extra right-moving degrees of freedom and reinstalls the balance of central charges: $c_{L}=c_{R}$. The Virasoro condition does not impose any new constraints, since the equation $\operatorname{tr} A^{2}=\operatorname{tr} B^{2}$ automatically holds for any element of $\mathfrak{h}_{2}$.

\section{A.4 Type- $U 4$}

The type- $U 4$ cosets are defined for $\operatorname{PSU}(n \mid n)$ with even $n$. We can also assume that $n>2$, as $\mathfrak{h}_{2}$ is empty for ${ }^{15} n=2$. The $\mathbb{Z}_{4}$ symmetry acts as

$$
\Omega \circ\left(\begin{array}{cc}
A & \Theta \\
\Psi & B
\end{array}\right)=\left(\begin{array}{cc}
J A^{t} J & -J \Psi^{t} J \\
J \Theta^{t} J & J B^{t} J
\end{array}\right),
$$

and leads to the $\mathbb{Z}_{4}$ decomposition

$$
\begin{aligned}
& \mathfrak{h}_{2}: A^{t}=-J A J, \quad B^{t}=-J B J \\
& \mathfrak{h}_{1}: \Psi=-i J \Theta^{t} J \\
& \mathfrak{h}_{3}: \Psi=i J \Theta^{t} J .
\end{aligned}
$$

The second equation in (A.2) is a consequence of the first for this coset. To find the rank of the kappa-symmetry, we just need to solve

$$
A \Theta=\Theta B .
$$

Non-trivial solutions to this equation correspond to pairs of equal eigenvalues of $A$ and $B$.

A matrix that satisfies the condition (A.27) can be represented as a product of two anti-symmetric matrices: $A=-(A J) J$, and consequently has doubly-degenerate spectrum, by Stenzel theorem. In addition, $A$ and $B$ are traceless and so have $n / 2-1$ independent eigenvalues. In general these eigenvalues have no reasons to coincide. Consequently, the coset has no extrinsic kappa-symmetries:

$$
\hat{N}_{\kappa}=\hat{N}_{\tilde{\kappa}}=0 .
$$

The Virasoro condition (A.3) imposes the relationship on the sums of squares of the eigenvalues of $A$ and $B$. In general this is not enough to force them to coincide. The only exception is the case of $n=4$, when $A$ and $B$ have just one independent eigenvalue each: $\{\alpha, \alpha-\alpha,-\alpha\}$ and $\{\beta, \beta,-\beta,-\beta\}$. The Virasoro condition means that $\alpha= \pm \beta$. Then (A.29) has an eight-dimensional space of solutions, and

$$
N_{\kappa}=N_{\tilde{\kappa}}=8 \quad(n=4)
$$

\footnotetext{
${ }^{15}$ Once can however consider the coset of $\mathrm{U}(2 \mid 2)$ (or even $\mathrm{U}(1 \mid 1)$ ) instead of $\operatorname{PSU}(2 \mid 2)$, then two bosonic directions survive the $\mathbb{Z}_{4}$ projection [39].
} 


\section{A.5 Type-O1}

Since $\Psi$ and $\Theta$ in $\mathfrak{o s p}(2 n+2 \mid 2 n)$ are related, for type- $O$ cosets we only need to solve one equation in (A.2):

$$
A \Theta=\Theta B
$$

the other will automatically follow.

The $\mathbb{Z}_{4}$ automorphism of the type-O1 cosets in the supermatrix representation (4.2) acts as follows:

$$
\Omega \circ\left(\begin{array}{cc}
A & \Theta \\
\Psi & B
\end{array}\right)=\left(\begin{array}{cc}
I_{p} A I_{p} & I_{p} \Theta J \\
-J \Psi I_{p} & -J B J
\end{array}\right)=\left(\begin{array}{cc}
I_{p} A I_{p} & I_{p} \Theta J \\
-J \Psi I_{p} & -B^{t}
\end{array}\right) .
$$

The associated $\mathbb{Z}_{4}$ decomposition is

$$
\begin{aligned}
& \mathfrak{h}_{2}: \quad A=\left(\begin{array}{cc}
0_{p \times p} & {\left[A_{1}\right]_{p \times(2 n+2-p)}} \\
-\left[A_{1}^{t}\right]_{(2 n+2-p) \times p} & 0_{(2 n+2-p) \times(2 n+2-p)}
\end{array}\right), \\
& B=\left(\begin{array}{ll}
{\left[B_{1}\right]_{n \times n}} & {\left[B_{2}\right]_{n \times n}} \\
{\left[B_{2}\right]_{n \times n}} & -\left[B_{1}\right]_{n \times n}
\end{array}\right), B_{i}^{t}=B_{i} \\
& \mathfrak{h}_{1}: \quad \Theta=\left(\begin{array}{cc}
{\left[\Theta_{1}\right]_{p \times n}} & -i\left[\Theta_{1}\right]_{p \times n} \\
{\left[\Theta_{2}\right]_{(2 n+2-p) \times n}} & i\left[\Theta_{2}\right]_{(2 n+2-p) \times n}
\end{array}\right) \\
& \mathfrak{h}_{3}: \quad \Theta=\left(\begin{array}{cc}
{\left[\Theta_{1}\right]_{p \times n}} & i\left[\Theta_{1}\right]_{p \times n} \\
{\left[\Theta_{2}\right]_{(2 n+2-p) \times n}} & -i\left[\Theta_{2}\right]_{(2 n+2-p) \times n}
\end{array}\right)
\end{aligned}
$$

The kappa-symmetry condition (A.32) in $\mathfrak{h}_{1 / 3}$ reduces to

$$
A_{1} \Theta_{2}=\Theta_{1} B_{\mp}, \quad-A_{1}^{t} \Theta_{1}=\Theta_{2} B_{ \pm},
$$

where

$$
B_{ \pm}=B_{1} \pm i B_{2} .
$$

Then $\Theta_{2}=-A_{1}^{t} \Theta_{1} B_{ \pm}^{-1}$, and we are left with the equation

$$
-A_{1} A_{1}^{t} \Theta_{1}=\Theta_{1} B_{\mp} B_{ \pm}
$$

for the $p \times n$ matrix $\Theta_{1}$. In general this equation has no solutions and, consequently, there will be no kappa-symmetries:

$$
\hat{N}_{\kappa}=\hat{N}_{\tilde{\kappa}}=0,
$$

because $-A_{1} A_{1}^{t}$ and $B_{\mp} B_{ \pm}$have different eigenvalues for generic matrices $A_{1}, B_{1}, B_{2}$. The null condition (A.3) relates the sums of the eigenvalues, because

$$
\operatorname{tr} A^{2}=-2 \operatorname{tr} A_{1} A_{1}^{t}, \quad \operatorname{tr} B^{2}=2 \operatorname{tr} B_{\mp} B_{ \pm} .
$$

This is still insufficient for the eigenvalues to coincide, except for the special case of $p=1$, $n=1$. Then both $-A_{1} A_{1}^{t}$ and $B_{\mp} B_{ \pm}$are numbers rather than matrices, which must coincide once the trace condition (A.3) is imposed. We thus find one solution in $\mathfrak{h}_{1}$ and one in $\mathfrak{h}_{3}$ :

$$
N_{\kappa}=N_{\tilde{\kappa}}=1 \quad(p=1, n=1) .
$$


There is also an extremely degenerate case of $p=0, n=1$. The target space then is $A d S_{2}$, without any extra factors. There are no propagating bosonic degrees of freedom. The number of kappa-symmetries, and consequently the number of fermionic degrees of freedom, depends on whether the string is left- or right-moving in the target space. In one case, there is no kappa-symmetries, and in the other case the kappa-symmetry removes all the fermions: $N_{\kappa}=4=N_{\tilde{\kappa}}$. The string then is purely topological.

\section{A.6 Type- $O 2$}

The $\mathbb{Z}_{4}$ automorphism in this case acts as

$$
\Omega \circ\left(\begin{array}{cc}
A & \Theta \\
\Psi & B
\end{array}\right)=\left(\begin{array}{cc}
-J A J & -J \Theta \mathbb{1} \otimes I_{p} \\
\mathbb{1} \otimes I_{p} \Psi J \mathbb{1} \otimes I_{p} B \mathbb{1} \otimes I_{p}
\end{array}\right) .
$$

It is convenient to work in the basis in which

$$
\mathbb{1} \otimes I_{p}=\left(\begin{array}{cc}
\mathbb{1}_{2 p \times 2 p} & 0 \\
0 & \mathbb{1}_{(2 n-2 p) \times(2 n-2 p)}
\end{array}\right) .
$$

The $\mathbb{Z}_{4}$ decomposition in this basis takes the form (we assume that $n-p \geqslant p$ ):

$$
\begin{aligned}
& \mathfrak{h}_{2}: \quad A=\left(\begin{array}{ll}
{\left[A_{1}\right]_{(n+1) \times(n+1)}} & {\left[A_{2}\right]_{(n+1) \times(n+1)}} \\
{\left[A_{2}\right]_{(n+1) \times(n+1)}} & -\left[A_{1}\right]_{(n+1) \times(n+1)}
\end{array}\right), A_{i}^{t}=-A_{i} \\
& B=\left(\begin{array}{cc}
0_{2 p \times 2 p} & {\left[B_{1}\right]_{2 p \times(2 n-2 p)}} \\
J_{(2 n-2 p) \times(2 n-2 p)}\left[B_{1}^{t}\right]_{(2 n-2 p) \times 2 p} J_{2 p \times 2 p} & 0_{(2 n-2 p) \times(2 n-2 p)}
\end{array}\right) \\
& \mathfrak{h}_{1}: \Theta=\left(\begin{array}{cc}
{\left[\Theta_{1}\right]_{(n+1) \times 2 p}} & {\left[\Theta_{2}\right]_{(n+1) \times(2 n-2 p)}} \\
-i\left[\Theta_{1}\right]_{(n+1) \times 2 p} & i\left[\Theta_{2}\right]_{(n+1) \times(2 n-2 p)}
\end{array}\right) \\
& \mathfrak{h}_{3}: \quad \Theta=\left(\begin{array}{cc}
{\left[\Theta_{1}\right]_{(n+1) \times 2 p}} & {\left[\Theta_{2}\right]_{(n+1) \times(2 n-2 p)}} \\
i\left[\Theta_{1}\right]_{(n+1) \times 2 p} & -i\left[\Theta_{2}\right]_{(n+1) \times(2 n-2 p)}
\end{array}\right)
\end{aligned}
$$

The zero-mode equation (A.32) in the $\mathfrak{h}_{1 / 3}$ subspace boils down to a system of two equations for matrices $\Theta_{1}, \Theta_{2}$ :

$$
\begin{aligned}
& A_{\mp} \Theta_{1}=\Theta_{2} J B_{1}^{t} J \\
& A_{ \pm} \Theta_{2}=\Theta_{1} B_{1},
\end{aligned}
$$

where

$$
A_{ \pm}=A_{1} \pm i A_{2}
$$

We need to distinguish even and odd $n$. Consider first odd $n$. The anti-symmetric matrices $A_{ \pm}$are then non-degenerate and we can express $\Theta_{2}$ through $\Theta_{1}: \Theta_{2}=A_{ \pm}^{-1} \Theta_{1} B_{1}$, substitute the result into the first equation and get:

$$
A_{ \pm} A_{\mp} \Theta_{1}=\Theta_{1} B_{1} J B_{1}^{t} J
$$

Both $A_{ \pm} A_{\mp}$ and $\left(B_{1} J B_{1}^{t}\right) J$ are products of two anti-symmetric matrices, their spectra are thus degenerate and contain, respectively, $(n+1) / 2$ and $p$ different eigenvalues: 
$\alpha_{1}, \alpha_{1}, \ldots, \alpha_{(n+1) / 2}, \alpha_{(n+1) / 2}$ and $\beta_{1}, \beta_{1}, \ldots \beta_{p}, \beta_{p}$. These eigenvalues are in general different. Hence there are no non-trivial solutions for $\Theta_{1}$, and there are no kappa-symmetries:

$$
\hat{N}_{\kappa}=\hat{N}_{\tilde{\kappa}}=0 \quad(n \text { odd })
$$

Imposing the Virasoro constraints does not change the situation, because the condition (A.3) imposes just one constraint on $\alpha_{i}, \beta_{j}$ :

$$
\sum_{i} \alpha_{i}=\sum_{j} \beta_{j}
$$

The only exception is the degenerate case of $n=1, p=0$, which is completely analogous to the type-O1 coset with $n=1, p=0$, discussed at the end of section A.5.

If $n$ is even, the matrix $A_{ \pm}$has one zero eigenvalue:

$$
A_{ \pm} v=0
$$

This gives $2 n-4 p$ solutions of (A.43), in combination with $2 n-4 p$ null eigenvalues of $J B_{1}^{t} J$ :

$$
u_{i} J B_{1}^{t} J=0, \quad i=1, \ldots 2 n-4 p .
$$

The solutions due to the null eigenvalues are:

$$
\Theta_{1}=0, \quad \Theta_{2}=v \otimes u_{i} .
$$

Potentially, there may also be solutions due to coincident non-zero eigenvalues of $A$ and $B$, which are given by the equation (A.45). This requires coincidence of some eigenvalues of $A_{ \pm} A_{ \pm}, \alpha_{1}, \alpha_{1}, \ldots, \alpha_{n / 2}, \alpha_{n / 2}, 0$, and $B_{1} J B_{1}^{t} J, \beta_{1}, \beta_{1}, \ldots, \beta_{p}, \beta_{p}$. In general, this does not happen, and thus

$$
\hat{N}_{\kappa}=2 n-4 p=\hat{N}_{\tilde{\kappa}} \quad(n \text { even }) .
$$

But if we impose the Virasoro condition, the eigenvalues satisfy (A.47), and for $n=2$, $p=1$, the matrices $A_{ \pm} A_{ \pm}$and $B_{1} J B_{1}^{t} J$ have two pairs of coinciding eigenvalues, leading to 4 extra solutions:

$$
N_{\kappa}=4=N_{\tilde{\kappa}} \quad(n=2, p=1) .
$$

\section{A.7 Type-Tu}

The $\mathbb{Z}_{4}$ generator of the the tensor-product models (4.6) acts on $\mathfrak{g}=\mathfrak{p} \oplus \mathfrak{p}$ as

$$
\Omega(X, Y)=\left(Y,(-1)^{F} \circ X\right)
$$

and gives the following $\mathbb{Z}_{4}$ decomposition:

$$
\begin{aligned}
& \mathfrak{h}_{2}:(X,-X), \quad X \in \mathfrak{p}_{\text {bos }} \\
& \mathfrak{h}_{1}:(\Xi,-i \Xi), \quad \Xi \in \mathfrak{p}_{\text {ferm }} \\
& \mathfrak{h}_{3}:(\Xi, i \Xi), \quad \Xi \in \mathfrak{p}_{\text {ferm }} .
\end{aligned}
$$


The kernel of ad $K, K=(X,-X) \in \mathfrak{h}_{2}$ in $\mathfrak{h}_{1 / 3}$ is determined by the equation

$$
([X, \Xi], \pm i[X, \Xi])=(0,0) \Longleftrightarrow[X, \Xi]=0,
$$

where $\Xi$ is an odd (fermionic) element of $\mathfrak{p}=\mathfrak{p s u}(n \mid n)$. This reduces to (A.2) for generic $n \times n$ matrices $\Theta$ and $\Psi$. In general, $A$ and $B$ have different eigenvalues, and there will be no solutions yielding

$$
\hat{N}_{\kappa}=0=\hat{N}_{\tilde{\kappa}} .
$$

The only exceptional case in which the null condition (A.3) makes a difference is $n=2$. Then there are two solutions for each of the matrices $\Theta$ and $\Psi$, and thus

$$
N_{\kappa}=N_{\tilde{\kappa}}=4 \quad(n=2) .
$$

\section{A.8 Type-To}

The matrices $A$ and $B$ in (A.2) never have common eigenvalues for $\mathfrak{p}=\mathfrak{o s p}(2 n+2 \mid 2 n)$, even if the null condition (A.3) is imposed. So,

$$
\hat{N}_{\kappa}=\hat{N}_{\tilde{\kappa}}=0,
$$

and there are no exceptional cases.

Open Access. This article is distributed under the terms of the Creative Commons Attribution Noncommercial License which permits any noncommercial use, distribution, and reproduction in any medium, provided the original author(s) and source are credited.

\section{References}

[1] V.V. Serganova, Classification of real simple Lie superalgebras and symmetric superspaces, Funkts. Anal. Prilozh. 17 (1983) 46 [Funct. Anal. Appl. 17 (1983) 200].

[2] N. Berkovits, M. Bershadsky, T. Hauer, S. Zhukov and B. Zwiebach, Superstring theory on $A d S_{2} \times S^{2}$ as a coset supermanifold, Nucl. Phys. B 567 (2000) 61 [hep-th/9907200] [SPIRES].

[3] R.R. Metsaev and A.A. Tseytlin, Type IIB superstring action in $A d S_{5} \times S^{5}$ background, Nucl. Phys. B 533 (1998) 109 [hep-th/9805028] [SPIRES].

[4] R. Roiban and W. Siegel, Superstrings on $A d S_{5} \times S^{5}$ supertwistor space, JHEP 11 (2000) 024 [hep-th/0010104] [SPIRES].

[5] I. Bena, J. Polchinski and R. Roiban, Hidden symmetries of the $A d S_{5} \times S^{5}$ superstring, Phys. Rev. D 69 (2004) 046002 [hep-th/0305116] [SPIRES].

[6] H. Eichenherr and M. Forger, On the dual symmetry of the nonlinear sigma models, Nucl. Phys. B 155 (1979) 381 [SPIRES].

[7] A.M. Polyakov, Interaction of Goldstone particles in two-dimensions. Applications to ferromagnets and massive Yang-Mills fields, Phys. Lett. B 59 (1975) 79 [SPIRES].

[8] A.M. Polyakov, Conformal fixed points of unidentified gauge theories, Mod. Phys. Lett. A 19 (2004) 1649 [hep-th/0405106] [SPIRES]. 
[9] I. Adam, A. Dekel, L. Mazzucato and Y. Oz, Integrability of type-II superstrings on Ramond-Ramond backgrounds in various dimensions, JHEP 06 (2007) 085 [hep-th/0702083] [SPIRES].

[10] M.B. Green and J.H. Schwarz, Covariant description of superstrings, Phys. Lett. B 136 (1984) 367 [SPIRES].

[11] J. Rahmfeld and A. Rajaraman, The GS string action on $A d S_{3} \times S^{3}$ with Ramond-Ramond charge, Phys. Rev. D 60 (1999) 064014 [hep-th/9809164] [SPIRES].

[12] J. Park and S.-J. Rey, Green-Schwarz superstring on $A d S_{3} \times S^{3}$, JHEP 01 (1999) 001 [hep-th/9812062] [SPIRES].

[13] J.-G. Zhou, Super 0-brane and GS superstring actions on $A d S_{2} \times S^{2}$, Nucl. Phys. B 559 (1999) 92 [hep-th/9906013] [SPIRES].

[14] R.R. Metsaev and A.A. Tseytlin, Superparticle and superstring in $A d S_{3} \times S^{3}$ Ramond-Ramond background in light-cone gauge, J. Math. Phys. 42 (2001) 2987 [hep-th/0011191] [SPIRES].

[15] H.L. Verlinde, Superstrings on $A d S_{2}$ and superconformal matrix quantum mechanics, hep-th/0403024 [SPIRES].

[16] B. Chen, Y.-L. He, P. Zhang and X.-C. Song, Flat currents of the Green-Schwarz superstrings in $A d S_{5} \times S^{1}$ and $A d S_{3} \times S^{3}$ backgrounds, Phys. Rev. D 71 (2005) 086007 [hep-th/0503089] [SPIRES].

[17] M. Hatsuda and Y. Michishita, Kappa symmetric OSp(2|2) WZNW model, JHEP 06 (2008) 049 [arXiv:0804.1831] [SPIRES].

[18] G. Arutyunov and S. Frolov, Superstrings on $A d S_{4} \times C P^{3}$ as a Coset $\sigma$-model, JHEP 09 (2008) 129 [arXiv:0806.4940] [SPIRES].

[19] B. Stefanski jr., Green-Schwarz action for Type IIA strings on $A d S_{4} \times C P^{3}$, Nucl. Phys. B 808 (2009) 80 [arXiv:0806.4948] [SPIRES].

[20] A. Babichenko, B. Stefanski jr. and K. Zarembo, Integrability and the $A d S_{3} / C F T_{2}$ correspondence, JHEP 03 (2010) 058 [arXiv:0912.1723] [SPIRES].

[21] B.C. Vallilo, One loop conformal invariance of the superstring in an $A d S_{5} \times S^{5}$ background, JHEP 12 (2002) 042 [hep-th/0210064] [SPIRES].

[22] D. Kagan and C.A.S. Young, Conformal $\sigma$-models on supercoset targets, Nucl. Phys. B $\mathbf{7 4 5}$ (2006) 109 [hep-th/0512250] [SPIRES].

[23] V.G.M. Puletti, Operator product expansion for pure spinor superstring on $A d S_{5} \times S^{5}$, JHEP 10 (2006) 057 [hep-th/0607076] [SPIRES].

[24] L. Mazzucato and B.C. Vallilo, On the non-renormalization of the AdS radius, JHEP 09 (2009) 056 [arXiv: 0906 . 4572] [SPIRES].

[25] S. Carlip, Heterotic string path integrals with the Green-Schwarz covariant action, Nucl. Phys. B 284 (1987) 365 [SPIRES].

[26] R. Kallosh and A.Y. Morozov, Green-Schwarz action and loop calculations for superstring, Int. J. Mod. Phys. A 3 (1988) 1943 [Sov. Phys. JETP 67 (1988) 1540] [Zh. Eksp. Teor. Fiz. 94N8 (1988) 42] [SPIRES].

[27] P.B. Wiegmann, Extrinsic geometry of superstrings, Nucl. Phys. B 323 (1989) 330 [SPIRES]. 
[28] N. Berkovits, C. Vafa and E. Witten, Conformal field theory of AdS background with Ramond-Ramond flux, JHEP 03 (1999) 018 [hep-th/9902098] [SPIRES].

[29] M. Bershadsky, S. Zhukov and A. Vaintrob, $\operatorname{PSL}(n \mid n) \sigma$-model as a conformal field theory, Nucl. Phys. B 559 (1999) 205 [hep-th/9902180] [SPIRES].

[30] N. Read and H. Saleur, Exact spectra of conformal supersymmetric nonlinear $\sigma$-models in two dimensions, Nucl. Phys. B 613 (2001) 409 [hep-th/0106124] [SPIRES].

[31] A. Babichenko, Conformal invariance and quantum integrability of $\sigma$-models on symmetric superspaces, Phys. Lett. B 648 (2007) 254 [hep-th/0611214] [SPIRES].

[32] V.G. Kac, A sketch of Lie superalgebra theory, Commun. Math. Phys. 53 (1977) 31 [SPIRES].

[33] L. Frappat, P. Sorba and A. Sciarrino, Dictionary on Lie superalgebras, hep-th/9607161 [SPIRES].

[34] U. Kraemmer and A. Rebhan, Anomalous anomalies in the Carlip-Kallosh quantization of the Green-Schwarz superstring, Phys. Lett. B 236 (1990) 255 [SPIRES].

[35] F. Bastianelli, P. van Nieuwenhuizen and A. Van Proeyen, Superstring anomalies in the semilight cone gauge, Phys. Lett. B 253 (1991) 67 [SPIRES].

[36] M. Porrati and P. van Nieuwenhuizen, Absence of world sheet and space-time anomalies in the semicovariantly quantized heterotic string, Phys. Lett. B 273 (1991) 47 [SPIRES].

[37] S. Bellucci and R.N. Oerter, Weyl invariance of the Green-Schwarz heterotic sigma model, Nucl. Phys. B 363 (1991) 573 [SPIRES].

[38] S.K. Ashok, R. Benichou and J. Troost, Conformal current algebra in two dimensions, JHEP 06 (2009) 017 [arXiv:0903.4277] [SPIRES].

[39] B. Stefanski jr., Landau-Lifshitz $\sigma$-models, fermions and the AdS/CFT correspondence, JHEP 07 (2007) 009 [arXiv:0704.1460] [SPIRES].

[40] J. Gomis, D. Sorokin and L. Wulff, The complete $A d S_{4} \times C P^{3}$ superspace for the type IIA superstring and D-branes, JHEP 03 (2009) 015 [arXiv:0811.1566] [SPIRES].

[41] I. Pesando, The GS type IIB superstring action on $A d S_{3} \times S^{3} \times T^{4}$, JHEP 02 (1999) 007 [hep-th/9809145] [SPIRES].

[42] C. Candu, V. Mitev, T. Quella, H. Saleur and V. Schomerus, The $\sigma$-model on complex projective superspaces, JHEP 02 (2010) 015 [arXiv:0908.0878] [SPIRES].

[43] C. Candu, T. Creutzig, V. Mitev and V. Schomerus, Cohomological reduction of $\sigma$-models, arXiv: 1001.1344 [SPIRES].

[44] V.A. Kazakov, A. Marshakov, J.A. Minahan and K. Zarembo, Classical/quantum integrability in AdS/CFT, JHEP 05 (2004) 024 [hep-th/0402207] [SPIRES].

[45] N. Beisert, V.A. Kazakov, K. Sakai and K. Zarembo, The algebraic curve of classical superstrings on $A d S_{5} \times S^{5}$, Commun. Math. Phys. 263 (2006) 659 [hep-th/0502226] [SPIRES].

[46] N. Gromov and P. Vieira, The AdS $S_{4} / C F T_{3}$ algebraic curve, JHEP 02 (2009) 040 [arXiv: 0807.0437] [SPIRES].

[47] N. Beisert, The SU(2|2) dynamic S-matrix, Adv. Theor. Math. Phys. 12 (2008) 945 [hep-th/0511082] [SPIRES]. 
[48] C. Ahn and R.I. Nepomechie, $N=6$ super Chern-Simons theory S-matrix and all-loop Bethe ansatz equations, JHEP 09 (2008) 010 [arXiv:0807.1924] [SPIRES].

[49] N. Beisert and M. Staudacher, Long-range PSU(2,2|4) Bethe ansätze for gauge theory and strings, Nucl. Phys. B 727 (2005) 1 [hep-th/0504190] [SPIRES].

[50] N. Gromov and P. Vieira, The all loop AdS $S_{4} / C F T_{3}$ Bethe ansatz, JHEP 01 (2009) 016 [arXiv: 0807.0777] [SPIRES].

[51] N. Gromov, V. Kazakov and P. Vieira, Exact spectrum of anomalous dimensions of planar $N=4$ supersymmetric Yang-Mills theory, Phys. Rev. Lett. 103 (2009) 131601 [arXiv: 0901.3753] [SPIRES].

[52] D. Bombardelli, D. Fioravanti and R. Tateo, Thermodynamic Bethe ansatz for planar AdS/CFT: a proposal, J. Phys. A 42 (2009) 375401 [arXiv:0902.3930] [SPIRES].

[53] N. Gromov, V. Kazakov, A. Kozak and P. Vieira, Exact spectrum of anomalous dimensions of planar $N=4$ supersymmetric Yang-Mills theory: TBA and excited states, Lett. Math. Phys. 91 (2010) 265 [arXiv:0902.4458] [SPIRES].

[54] G. Arutyunov and S. Frolov, Thermodynamic Bethe ansatz for the AdS $S_{5} \times S^{5}$ mirror model, JHEP 05 (2009) 068 [arXiv:0903.0141] [SPIRES].

[55] D. Bombardelli, D. Fioravanti and R. Tateo, TBA and $Y$-system for planar $A d S_{4} / C F T_{3}$, Nucl. Phys. B 834 (2010) 543 [arXiv: 0912.4715] [SPIRES].

[56] N. Gromov and F. Levkovich-Maslyuk, Y-system, TBA and quasi-classical strings in $A d S_{4} \times C P^{3}$, arXiv:0912.4911 [SPIRES].

[57] H. Stenzel, Über die Darstellbarkeit einer Matrix als Produkt von zwei symmetrischer Matrizen, als Produkt von zwei alternierenden Matrizen und als Produkt von einer symmetrischen und einer alternierenden Matrix (in German), Math. Z. 15 (1922) 1.

[58] K.D. Ikramov and H. Fassbender, On the product of two skew-Hamiltonian matrices or two skew-symmetric matrices, J. Math. Sci. 157 (2009) 697 [Zap. Nauchn. Sem. POMI 359 (2008) 45]. 